\title{
Chitosan-silica Sulfate Nano Hybrid: An Efficient Biopolymer Based-heterogeneous Nano Catalyst for Solvent-free Synthesis of 3,4-Dihydropyrimidine-2(1H)-one/thiones
}

\author{
Somayeh Behrouz, ${ }^{1, \star}$ Masoome Nazar Abi ${ }^{1}$ and Mohammad Amin Piltan ${ }^{1}$ \\ ${ }^{1}$ Medicinal Chemistry Research Laboratory, Department of Chemistry, Shiraz University of Technology, \\ Shiraz 71555-313, Iran \\ *Corresponding author: E-mail: behrouz@sutech.ac.ir \\ phone: +98-713-7354520; fax: +98-713-7354523
}

Received: 08-20-2020

\begin{abstract}
A green and highly efficient approach for the synthesis of 3,4-dihydropyrimidine-2(1H)-one/thione derivatives is described. In this approach, the three-component Biginelli reaction between (thio)urea, methyl acetoacetate and aldehydes under solvent-free condition in the presence of chitosan-silica sulfate nano hybrid (CSSNH) as a green and heterogeneous nano catalyst affords the corresponding products in good to excellent yields and in short reaction times. CSSNH is a cheap, eco-friendly, and non-toxic nano catalyst that could be easily prepared, handled, and reused for many reaction runs without significant loss of its activity.
\end{abstract}

Keywords: Chitosan-silica sulfate nano hybrid; 3,4-Dihydropyrimidines; green chemistry; heterogeneous catalysis; multicomponent reaction

\section{Introduction}

In recent decades, the status of environmental issues has dramatically directed the main goal of science and technology towards environmentally benign processes. Among different tasks to achieve this target, replacing the harmful solvents/catalysts with the green solvents/cata- lysts and even performing the reactions in the absence of solvent are of high significance. ${ }^{1,2}$ Meanwhile, multi-component reactions (MCRs) have gained tremendous attention in comparison with established stepwise synthesis since MCRs exhibit various significant advantages which are in accordance with the green chemistry guidelines and protocols. ${ }^{2,3}$ MCRs afford desirable benefits such as at-<smiles>CCOC(=O)C1=C(C)NC(=S)NC1c1cccc(O)c1</smiles>

Monastrol, anticancer<smiles>CCCOC(=O)C1=C(C)NC(=O)NC1c1ccc([N+](=O)[O-])cc1</smiles>

antibacterial<smiles>CCOC(=O)C1=C(C)NC(=S)NC1c1ccc2c(c1)OCO2</smiles>

Piperastrol, anticancer<smiles>CCC1=C(C(=O)OCC2CCCCC2)C(c2ccc(Cl)cc2)NC(=O)N1</smiles>
anti-HIV<smiles>CCCOC(=O)C1=C(C)N(C(=O)NCc2ccccc2)C(=O)NC1c1cccc([N+](=O)[O-])c1</smiles>

calcium channel inhibitor<smiles>COC(=O)C1=C(C)NC(=O)NC1c1ccc(Br)c([N+](=O)[O-])c1</smiles>

anti-HSV<smiles>CCOC(=O)C1=C(C)NC(=O)NC1c1cc(Cl)ccc1O</smiles>

anti-inflammatory<smiles>CCOC(=O)C1=C(C)NC(=O)NC1c1cc(OC)c(O)c(OC)c1</smiles>

Figure 1. Structure and activity of some DHPMs. 
om-efficiency, minimizing the waste and pollution, lowcost and green transformations, simple operation, and high yields. ${ }^{2,3}$ MCRs prove to be an ideal strategy for easy and quick access to numerous heterocyclic compounds among which 3,4-dihydropyrimidine-2( $1 H)$-ones (DHPMs) derivatives have gained noticeable attention due to their unique and promising biological profiles. ${ }^{4,5}$ DHPMs display a wide spectrum of biological activities such as antidiabetic, anti-inflammatory, anticancer, antimalarial, antiviral, antiproliferative, antileishmanial, antihypertensive, calcium channel modulators and antibacterial in particular antitubercular. ${ }^{4,6}$ In addition, DHPMs have been widely used in polymer industries, fabric dyes, and adhesives. ${ }^{4}$ They are also found as natural alkaloids in marine creatures. ${ }^{7,8}$ The structure and the activity of several bioactive DHPMs are shown in Figure 1.,6

The rapid and straightforward method to access 3,4-dihydropyrimidine-2(1H)-ones/thiones involves the three-component cyclocondensation of aldehyde, $\beta$-ketoester, and (thio)urea under acidic condition namely known as Biginelli reaction. ${ }^{4,9}$ Due to the problems associated with the classical Biginelli reaction such as harsh reaction conditions, low yields, and long reaction times and also regarding to the significance of DHPMs, hence the numerous reaction conditions using various Lewis and Brønsted acid catalysts have been developed so far. ${ }^{5}$ To date, a plenty of homogeneous and heterogeneous catalysts such as nano $\mathrm{BF}_{3} \cdot \mathrm{SiO}_{2},{ }^{10} \mathrm{Bi}(\mathrm{III})$ supported on silica-coated $\mathrm{Fe}_{3} \mathrm{O}_{4}$ nanoparticles, ${ }^{1} \mathrm{TiCl}_{3} \mathrm{OTf}-[\mathrm{bmim}] \mathrm{Cl},{ }^{12}$ [TEAPS] $\mathrm{H}_{2} \mathrm{PMo}_{12} \mathrm{O}_{40},{ }^{13} \mathrm{CuCl}_{2} / \mathrm{HCl},{ }^{14}$ sulfated silica tungstic acid, ${ }^{15} \mathrm{ErCl}_{3} \cdot 6 \mathrm{H}_{2} \mathrm{O},{ }^{16} \mathrm{SnCl}_{2} /$ nano $\mathrm{SiO}_{2},{ }^{17} \mathrm{~L}$-proline nitrate, ${ }^{18}$ D-xylonic acid, ${ }^{19} \mathrm{Fe}(\mathrm{OTs}){ }_{3} \cdot 6 \mathrm{H}_{2} \mathrm{O},{ }^{20} \mathrm{NiCl}_{2} \cdot 6 \mathrm{H}_{2} \mathrm{O} /$ $\mathrm{HCl},{ }^{21}$ Co@imine- $\mathrm{Na}^{+}$-montmorillonite, ${ }^{22}$ dendrimer-attached phosphotungstic acid nanoparticles immobilized on nanosilica, ${ }^{23} \mathrm{Ce}(\mathrm{LS})_{3},{ }^{24}$ bentonite $/ \mathrm{PS}-\mathrm{SO}_{3} \mathrm{H},{ }^{25}$ silica sulfuric acid, ${ }^{26} \mathrm{HClO}_{4} \cdot \mathrm{SiO}_{2},{ }^{27}$ and the exchanged cations in an Algerian montmorillonite ${ }^{28}$ under conventional heating, ultrasound and microwave irradiations as well as solvent-free conditions have been reported to achieve DHPMs synthesis. ${ }^{5}$ Although these protocols are accompa- nied with several advantages; however, they suffer from several defects such as the use of metal-based, corrosive, toxic, expensive, non-reusable, and moisture sensitive catalysts, tedious work-up and purification processes, environmental contaminations, the use of harmful organic solvents, inadequate yields, and also long reaction times. Hence, their exploitation causes both economic and environmental concerns. In addition, the use of strongly acidic conditions has no compatibility with acid-sensitive moieties. Consequently, developing an alternative, mild and green protocol for synthesis of DHPMs which overcomes those limitations is still a challenging issue in organic chemistry.

Undoubtedly, catalysts play a crucial role in countless chemical processes. Taking the numerous advantages of heterogeneous catalysts from both environmental and economic points of view, the use of heterogeneous catalysts has attracted a massive attention in comparison with homogeneous catalysts from both academic and industrial aspects. Currently, the application of natural biopolymers is growing tremendously in different research areas especially for preparation of green heterogeneous catalysts owing to their biodegradable, biocompatible, non-toxic, and cheap materials. To this end, polysaccharides have found increasing applications since these biomolecules exhibit unique chelating power and ease of chemical modifications and also due to their abundancy in the nature. ${ }^{29}$ Along this line, utilizing the natural biopolymer-based catalysts for synthesis of DHPMs is an attractive strategy. In this context, the use of acidic heterogeneous catalysts including cellulose sulfuric acid ${ }^{30}$ and starch sulfuric acid ${ }^{31}$ has been reported in refluxing water and $\mathrm{EtOH}$, respectively. Lal et al. reported the synthesis of DHPM derivatives of curcumin using chitosan $/ \mathrm{AcOH} / \mathrm{H}_{2} \mathrm{O}{ }^{32}$ From both economic and ecological perspectives, utilizing the green nano heterogeneous catalysts under solvent-free condition is in demand. To the best of our knowledge, only one solvent-free synthesis of DHPMs using chitosan/ graphene oxide nanocomposite has been established. ${ }^{33}$ Hence, developing an efficient solvent-free protocol for

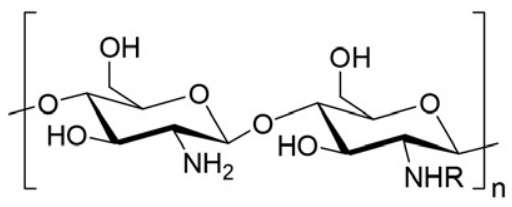

$\mathrm{R}=\mathrm{H}, \mathrm{Ac}$ cs

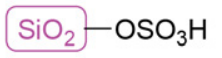

SSA

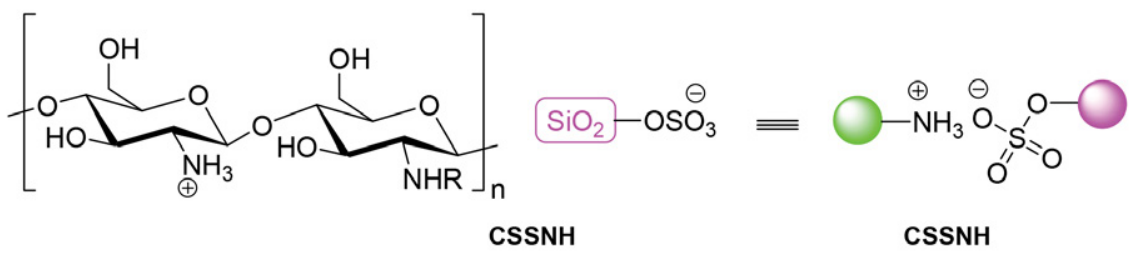

Figure 2. The structure of chitosan (CS), silica sulfuric acid (SSA) and CSSNH. 


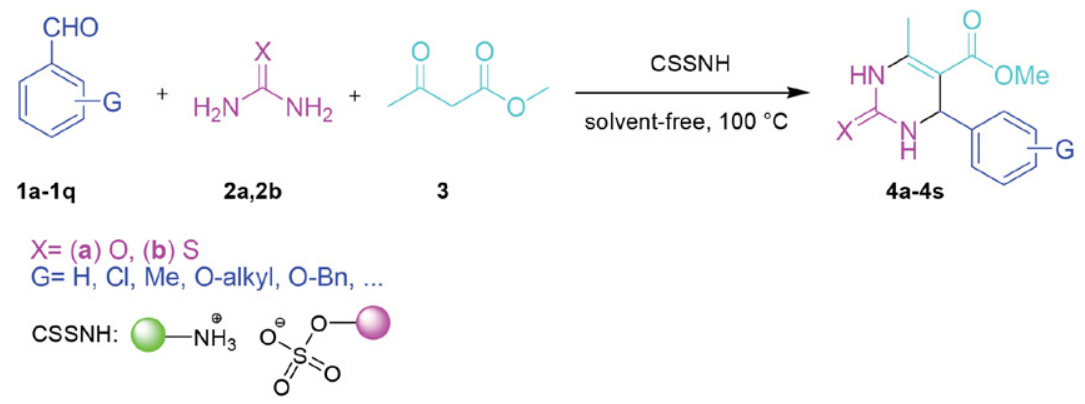

Scheme 1. Synthesis of DHPMs using CSSNH.

synthesis of DHPMs in the presence of biopolymer-based nano heterogeneous catalysts is still quite appealing.

Chitosan is a biodegradable, non-toxic, and cheap natural biopolymer which can easily undergo chemical modifications through its amine and hydroxyl functional groups. ${ }^{34}$ Accordingly, chitosan has found considerable research interest in numerous fields of science and industry. ${ }^{35-38}$ Recently, we have reported the synthesis, characterization, and application of chitosan-silica sulfate nanohybrid (CSSNH) as a new, green, highly proficient, and cheap heterogeneous nano-catalyst (Figure 2). The high efficacy and potency of CSSNH was confirmed by its successful applications in many organic transformations. ${ }^{39-41}$ Inspired by the unique biological profile of $\mathrm{DH}$ PMs and our interest in multi-component reactions ${ }^{42-46}$ as well as discovering another new application for CSSNH, hereby we report a facile and efficient protocol for synthesis of 3,4-dihydropyrimidine-2 $(1 H)$-one/thione derivatives via three-component reaction (3CR) of commercial and synthetic aldehydes, (thio)urea, and methyl acetoacetate in the presence of CSSNH as an eco-friendly and heterogeneous nano catalyst at $100{ }^{\circ} \mathrm{C}$ under the solvent-free condition (Scheme 1).

\section{Experimental}

All chemicals were purchased from Merck or Sigma-Aldrich. CSSNH was prepared according to the reported procedure. ${ }^{40}$ Solvents were purified by standard procedures, and stored over $3 \AA$ molecular sieves. Reactions were followed by TLC using SILG/UV 254 silica-gel plates. Column chromatography was performed on silica gel $60\left(0.063-0.200 \mathrm{~mm}, 70-230 \mathrm{mesh}\right.$; ASTM). ${ }^{1} \mathrm{H}$ and ${ }^{13} \mathrm{C}$ NMR spectra were recorded on Bruker AvanceDPX-300 spectrometer operating at $300 \mathrm{MHz}$ or $75 \mathrm{MHz}$, respectively. Chemical shifts are given in $\delta$ (on ppm scale) relative to tetramethylsilane (TMS) as an internal standard, coupling constants $J$ are given in Hz. HRMS and IR spectra were obtained using a Bruker micrOTOF-Q 134 apparatus and a Shimadzu FT-IR-8300 spectrophotometer, respectively. Melting points were measured using Electrothermal IA 9000 melting point apparatus in open capillary tubes and are uncorrected.
Preparation of 3,4-Dihydropyrimidine-2(1H)-(thio)ones
4a-s
In a round bottom flask $(25 \mathrm{~mL})$, a mixture of thio(urea) $(6 \mathrm{mmol})$, methyl acetoacetate $(5 \mathrm{mmol})$, appropriate aldehyde $(5 \mathrm{mmol})$, and CSSNH $(0.04 \mathrm{~g})$ under solvent free condition was heated at $100{ }^{\circ} \mathrm{C}$ in a paraffin bath. When no further improvement was observed on the reaction progress (TLC monitoring, Table 4), the reaction mixture was diluted with $\mathrm{EtOH}(10 \mathrm{~mL})$. Subsequently, the crude mixture was filtered off to separate the catalyst. The catalyst was then washed with $\mathrm{EtOH}(2 \times 5 \mathrm{~mL})$ to remove any substrate from CSSNH. The pure DHPM derivatives were obtained via recrystallization from the ethanolic solution of the reaction mixture.

\section{Recovery of CSSNH}

After accomplishment of the reaction and separation of CSSNH, the catalyst was washed with EtOH $(2 \times 5 \mathrm{~mL})$. $\mathrm{CSSNH}$ was then dried in a vacuum oven at $50{ }^{\circ} \mathrm{C}$ for $2 \mathrm{~h}$ and kept in a closed vessel in the refrigerator.

Methyl 6-Methyl-2-oxo-4-phenyl-1,2,3,4-tetrahydropyrimidine-5-carboxylate (4a) $\mathrm{C}_{13} \mathrm{H}_{14} \mathrm{~N}_{2} \mathrm{O}_{3}$

Recrystallization from ethanol afforded $1.18 \mathrm{~g}(96 \%)$ 4a as a white solid. Mp 208-210 ${ }^{\circ} \mathrm{C}$ (Lit. $\left..^{26} 210-212{ }^{\circ} \mathrm{C}\right) ;{ }^{1} \mathrm{H}$ NMR (300 MHz, DMSO- $\left.d_{6}\right): \delta 2.24\left(\mathrm{~s}, 3 \mathrm{H}, \mathrm{CH}_{3}\right), 3.51(\mathrm{~s}$, $\left.3 \mathrm{H}, \mathrm{OCH}_{3}\right), 5.12(\mathrm{~s}, 1 \mathrm{H}, \mathrm{PhCH}), 7.21-7.33(\mathrm{~m}, 5 \mathrm{H}, \mathrm{ArH})$, $7.76(\mathrm{~s}, 1 \mathrm{H}, \mathrm{NH}), 9.23(\mathrm{~s}, 1 \mathrm{H}, \mathrm{NH}) ;{ }^{13} \mathrm{C}$ NMR $(75 \mathrm{MHz}$, DMSO- $\left.d_{6}\right): \delta 17.89,50.87,53.85,99.03,126.04,127.22$, $128.42,144.67,148.58,152.14,165.88 ; \mathrm{IR}(\mathrm{KBr}): v_{\max } 3350$, 3180, 2950, 1700, 1640,1470, 1250, $1172 \mathrm{~cm}^{-1}$; HRMS: $m / z(\mathrm{M}+\mathrm{H})^{+}$calculated for $\mathrm{C}_{13} \mathrm{H}_{14} \mathrm{~N}_{2} \mathrm{O}_{3}: 247.1083$, found: 247.1097.

Methyl 6-Methyl-2-oxo-4-para-tolyl-1,2,3,4-tetrahydropyrimidine-5-carboxylate (4b) $\mathrm{C}_{14} \mathrm{H}_{16} \mathrm{~N}_{2} \mathrm{O}_{3}$

Recrystallization from ethanol afforded $1.22 \mathrm{~g}(94 \%)$ 4b as a white solid. Mp 199-201 ${ }^{\circ} \mathrm{C}$ (Lit. $\left.{ }^{49} 198-200{ }^{\circ} \mathrm{C}\right) ;{ }^{1} \mathrm{H}$ NMR (300 MHz, DMSO- $d_{6}$ ): $\delta$ 2.23-2.24 (Complex, 6H, $\left.\mathrm{CH}_{3}, \mathrm{PhCH}_{3}\right), 3.50\left(\mathrm{~s}, 3 \mathrm{H}, \mathrm{OCH}_{3}\right), 5.08(\mathrm{~s}, 1 \mathrm{H}, \mathrm{PhCH})$, 7.10 (br s, $4 \mathrm{H}, \mathrm{ArH}), 7.71$ (s, 1H, NH), 9.19 (s, 1H, NH); ${ }^{13} \mathrm{C}$ NMR $\left(75 \mathrm{MHz}, \mathrm{DMSO}-d_{6}\right): \delta 17.84,20.62,50.74$, $53.45,99.05,126.02,128.86,136.41,144.63,148.45,152.05$, 165.79; IR (KBr): $v_{\max } 3250,3125,2964,1698,1629,1438$, 
1257, $1172 \mathrm{~cm}^{-1}$; HRMS: $\mathrm{m} / z(\mathrm{M}+\mathrm{H})^{+}$calculated for $\mathrm{C}_{14} \mathrm{H}_{16} \mathrm{~N}_{2} \mathrm{O}_{3}: 261.1239$, found: 261.1252 .

Methyl 4-(2-Chlorophenyl)-6-methyl-2-oxo-1,2,3,4-tetrahydropyrimidine-5-carboxylate (4c) $\mathrm{C}_{13} \mathrm{H}_{13} \mathrm{ClN}_{2} \mathrm{O}_{3}$

Recrystallization from ethanol afforded $1.23 \mathrm{~g}(88 \%)$ 4c as a white solid. Mp 257-259 ${ }^{\circ} \mathrm{C}\left(\right.$ Lit. $\left.^{49}{ }^{256-259}{ }^{\circ} \mathrm{C}\right) ;{ }^{1} \mathrm{H}$ NMR $\left(300 \mathrm{MHz}, \mathrm{DMSO}-d_{6}\right): \delta 2.28\left(\mathrm{~s}, 3 \mathrm{H}, \mathrm{CH}_{3}\right), 3.43(\mathrm{~s}$, $\left.3 \mathrm{H}, \mathrm{OCH}_{3}\right), 5.43(\mathrm{~s}, 1 \mathrm{H}, \mathrm{PhCH}), 7.26-7.40(\mathrm{~m}, 4 \mathrm{H}, \mathrm{ArH})$, $7.72(\mathrm{~s}, 1 \mathrm{H}, \mathrm{NH}), 9.32(\mathrm{~s}, 1 \mathrm{H}, \mathrm{NH}) ;{ }^{13} \mathrm{C}$ NMR $(75 \mathrm{MHz}$, DMSO- $\left.d_{6}\right): \delta 17.86,51.97,59.58,98.40,128.16,129.09$, $129.23,129.87,132.14,142.25,149.83,151.79,165.55$; IR $(\mathrm{KBr}): v_{\max } 3240,3163,2975,1696,1620,1468,1225,1145$, $748 \mathrm{~cm}^{-1}$; HRMS: $\mathrm{m} / z(\mathrm{M}+\mathrm{H})^{+}$calculated for $\mathrm{C}_{13} \mathrm{H}_{13} \mathrm{Cl}-$ $\mathrm{N}_{2} \mathrm{O}_{3}: 281.0693$, found: 281.0715 .

Methyl 4-(2,4-Dichlorophenyl)-6-methyl-2-thioxo-1,2, 3,4-tetrahydropyrimidine-5-carboxylate (4d) $\mathrm{C}_{13} \mathrm{H}_{12}$ $\mathrm{Cl}_{2} \mathrm{~N}_{2} \mathrm{O}_{2} \mathrm{~S}$

Recrystallization from ethanol afforded $1.42 \mathrm{~g}(86 \%)$ 4d as a white solid. Mp 252-254 ${ }^{\circ} \mathrm{C}$; ${ }^{1} \mathrm{H}$ NMR $(300 \mathrm{MHz}$, DMSO- $\left.d_{6}\right): \delta 2.49\left(\mathrm{~s}, 3 \mathrm{H}, \mathrm{CH}_{3}\right), 3.35\left(\mathrm{~s}, 3 \mathrm{H}, \mathrm{OCH}_{3}\right), 5.43$ (s, 1H, PhCH), 6.99 (br s, 2H, ArH), 7.17 (s, 1H, ArH), 7.29 (s, 1H, NH), $9.00(\mathrm{~s}, 1 \mathrm{H}, \mathrm{NH}) ;{ }^{13} \mathrm{C}$ NMR $(75 \mathrm{MHz}$, DMSO- $\left.d_{6}\right): \delta 17.78,50.77,51.52,97.36,128.09,128.86$, 130.02, 132.46, 132.62, 140.90, 149.63, 151.25, 165.30; IR $(\mathrm{KBr}): v_{\max } 3340,3150,2961,1680,1459,1225,1190,1145$, $752 \mathrm{~cm}^{-1}$; HRMS: $\mathrm{m} / z(\mathrm{M}+\mathrm{H})^{+}$calculated for $\mathrm{C}_{13} \mathrm{H}_{12} \mathrm{Cl}-$ ${ }_{2} \mathrm{~N}_{2} \mathrm{O}_{2} \mathrm{~S}: 329.9997$, found: 330.0018 .

Methyl 4-(4-Methoxyphenyl)-6-methyl-2-oxo-1,2,3,4tetrahydropyrimidine-5-carboxylate $(4 \mathrm{e}) \mathrm{C}_{14} \mathrm{H}_{16} \mathrm{~N}_{2} \mathrm{O}_{4}$

Recrystallization from ethanol afforded $1.28 \mathrm{~g}(93 \%)$ 4e as a white solid. Mp $186-188^{\circ} \mathrm{C}\left(\mathrm{Lit}^{49}{ }^{4} 187-190{ }^{\circ} \mathrm{C}\right) ;{ }^{1} \mathrm{H}$ NMR (300 MHz, DMSO- $\left.d_{6}\right): \delta 2.21\left(\mathrm{~s}, 3 \mathrm{H}, \mathrm{CH}_{3}\right), 3.50(\mathrm{~s}$, $\left.3 \mathrm{H}, \mathrm{OCH}_{3}\right), 3.69\left(\mathrm{~s}, 3 \mathrm{H}, \mathrm{OCH}_{3}\right), 5.05(\mathrm{~s}, 1 \mathrm{H}, \mathrm{PhCH}), 6.85$ (br s, 2H, ArH), 7.11 (br s, 2H, ArH), $7.68(\mathrm{~s}, 1 \mathrm{H}, \mathrm{NH})$, $9.17(\mathrm{~s}, 1 \mathrm{H}, \mathrm{NH}) ;{ }^{13} \mathrm{C}$ NMR $\left(75 \mathrm{MHz}, \mathrm{DMSO}-d_{6}\right): \delta 17.86$, 50.64, 53.13, 54.99, 99.33, 113.73, 127.26, 136.83, 148.39, $152.19,158.48,165.84$; IR (KBr): $v_{\max } 3300,3170,2981$, 1693, 1618, 1455, 1237, $1148 \mathrm{~cm}^{-1}$; HRMS: $m / z(\mathrm{M}+\mathrm{H})^{+}$ calculated for $\mathrm{C}_{14} \mathrm{H}_{16} \mathrm{~N}_{2} \mathrm{O}_{4}$ : 277.1188, found: 277.1213.

Methyl 4-(4-Methoxyphenyl)-6-methyl-2-thioxo-1,2,3, 4-tetrahydropyrimidine-5-carboxylate (4f) $\mathrm{C}_{14} \mathrm{H}_{16} \mathrm{~N}_{2} \mathrm{O}_{3} \mathrm{~S}$ Recrystallization from ethanol afforded $1.33 \mathrm{~g}(91 \%)$ 4f as a yellow solid. Mp $178-180{ }^{\circ} \mathrm{C}\left(\right.$ Lit. $\left.^{18} 181-183^{\circ} \mathrm{C}\right) ;{ }^{1} \mathrm{H}$ NMR $\left(300 \mathrm{MHz}\right.$, DMSO- $\left.d_{6}\right): \delta 2.27\left(\mathrm{~s}, 3 \mathrm{H}, \mathrm{CH}_{3}\right), 3.53(\mathrm{~s}$, $\left.3 \mathrm{H}, \mathrm{OCH}_{3}\right), 3.71\left(\mathrm{~s}, 3 \mathrm{H}, \mathrm{OCH}_{3}\right), 5.09(\mathrm{~s}, 1 \mathrm{H}, \mathrm{PhCH}), 6.87$ $(\mathrm{d}, 2 \mathrm{H}, J=6.0 \mathrm{~Hz}, \mathrm{ArH}), 7.10(\mathrm{~d}, 2 \mathrm{H}, J=6.0 \mathrm{~Hz}, \mathrm{ArH}), 9.63$ (s, 1H, NH), 10.33 (s, 1H, NH); ${ }^{13} \mathrm{C}$ NMR $(75 \mathrm{MHz}$, DMSO- $\left.d_{6}\right): \delta 17.13,51.15,53.32,55.16,100.69,114.03$, 127.66, 135.51, 145.01, 158.79, 165.69, 174.12; IR (KBr): $v_{\max } 3310,3175,2943,1682,1439,1225,1190,1153 \mathrm{~cm}^{-1}$; HRMS: $m / z(\mathrm{M}+\mathrm{H})^{+}$calculated for $\mathrm{C}_{14} \mathrm{H}_{16} \mathrm{~N}_{2} \mathrm{O}_{3} \mathrm{~S}$ : 293.0960, found: 293.0974 .
Methyl 4-(3,5-Dimethoxyphenyl)-6-methyl-2-thioxo-1, 2,3,4-tetrahydropyrimidine-5-carboxylate $(4 \mathrm{~g}) \mathrm{C}_{15} \mathrm{H}_{18^{-}}$ $\mathrm{N}_{2} \mathrm{O}_{4} \mathrm{~S}$

Recrystallization from ethanol afforded $1.43 \mathrm{~g}(89 \%)$ 4g as a yellow solid. Mp $195-197^{\circ} \mathrm{C} ;{ }^{1} \mathrm{H}$ NMR $(300 \mathrm{MHz}$, DMSO- $\left.d_{6}\right): \delta 2.30\left(\mathrm{~s}, 3 \mathrm{H}, \mathrm{CH}_{3}\right), 3.57\left(\mathrm{~s}, 3 \mathrm{H}, \mathrm{OCH}_{3}\right), 3.73$ (s, 6H, $\left.2 \mathrm{OCH}_{3}\right), 5.14(\mathrm{~s}, 1 \mathrm{H}, \mathrm{PhCH}), 6.69-6.72(\mathrm{~m}, 1 \mathrm{H}$, ArH), 6.86-6.92 (m, 1H, ArH), $9.63(\mathrm{~s}, 1 \mathrm{H}, \mathrm{NH}), 10.34(\mathrm{~s}$, $1 \mathrm{H}, \mathrm{NH}) ;{ }^{13} \mathrm{C}$ NMR $\left(75 \mathrm{MHz}, \mathrm{DMSO}-d_{6}\right): \delta 17.26,51.15$, $53.45,55.34,100.11,101.11,113.96,136.21,145.63,159.08$, 165.83, 173.99; IR (KBr): $v_{\max } 3300,3193,2971,1685$, 1467, 1229, 1182, $1138 \mathrm{~cm}^{-1}$; HRMS: $m / z(\mathrm{M}+\mathrm{H})^{+}$calculated for $\mathrm{C}_{15} \mathrm{H}_{18} \mathrm{~N}_{2} \mathrm{O}_{4} \mathrm{~S}: 323.1066$, found: 323.1085 .

Methyl 4-(4-(5-(Methoxycarbonyl)-6-methyl-2-oxo-1,2, 3,4-tetrahydropyrimidin-4-yl)phenyl)-6-methyl-2oxo-1,2,3,4-tetrahydropyrimidine-5-carboxylate $\mathrm{C}_{20} \mathrm{H}_{22} \mathrm{~N}_{4} \mathrm{O}_{6}$

Recrystallization from ethanol afforded $1.80 \mathrm{~g}(87 \%)$ 4h as a white solid. $\mathrm{Mp}>245{ }^{\circ} \mathrm{C}$ (dec.); ${ }^{1} \mathrm{H}$ NMR (300 $\left.\mathrm{MHz}, \mathrm{DMSO}-d_{6}\right): \delta 2.51\left(\mathrm{~s}, 6 \mathrm{H}, 2 \mathrm{CH}_{3}\right), 3.36(\mathrm{~s}, 6 \mathrm{H}, 2$ $\mathrm{OCH}_{3}$ ), 5.65 (s, 2H, $2 \mathrm{PhCH}$ ), 7.18 (br s, $\left.4 \mathrm{H}, \mathrm{ArH}\right), 7.71$ (s, $2 \mathrm{H}, 2 \mathrm{NH}), 9.24(\mathrm{~s}, 2 \mathrm{H}, 2 \mathrm{NH}) ;{ }^{13} \mathrm{C}$ NMR $(75 \mathrm{MHz}$, DMSO- $\left.d_{6}\right): \delta 18.01,51.04,54.08,99.29,127.70,144.53$, 148.50, 152.12, 165.92; IR (KBr): $v_{\max } 3350,3240,3056$, 1700, 1645, 1472, 1250, $1140 \mathrm{~cm}^{-1}$; HRMS: $\mathrm{m} / z(\mathrm{M}+\mathrm{H})^{+}$ calculated for $\mathrm{C}_{20} \mathrm{H}_{22} \mathrm{~N}_{4} \mathrm{O}_{6}$ : 415.1618, found: 415.1641.

Methyl 4-(4-Butoxyphenyl)-6-methyl-2-oxo-1,2,3,4-tetrahydropyrimidine-5-carboxylate $(4 \mathrm{i}) \mathrm{C}_{17} \mathrm{H}_{22} \mathrm{~N}_{2} \mathrm{O}_{4}$

Recrystallization from ethanol afforded $1.46 \mathrm{~g}(92 \%)$ $4 \mathbf{i}$ as a white solid. Mp $173-175^{\circ} \mathrm{C}$; ${ }^{1} \mathrm{H}$ NMR $(300 \mathrm{MHz}$, DMSO- $\left.d_{6}\right): \delta 0.88\left(\mathrm{t}, 3 \mathrm{H}, J=6.0 \mathrm{~Hz}, \mathrm{CH}_{2} \mathrm{CH}_{3}\right), 1.39-1.41$ (m, $2 \mathrm{H}, \mathrm{CH}_{2} \mathrm{CH}_{3}$ ), 1.65 (br s, $\left.2 \mathrm{H}, \mathrm{OCH}_{2} \mathrm{CH}_{2}\right), 2.22(\mathrm{~s}, 3 \mathrm{H}$, $\left.\mathrm{CH}_{3}\right), 3.51\left(\mathrm{~s}, 3 \mathrm{H}, \mathrm{OCH}_{3}\right), 3.91\left(\mathrm{~s}, 2 \mathrm{H}, \mathrm{OCH}_{2}\right), 5.06(\mathrm{~s}, 1 \mathrm{H}$, $\mathrm{PhCH}), 6.84(\mathrm{~d}, 2 \mathrm{H}, J=3.0 \mathrm{~Hz}, \mathrm{ArH}), 7.09(\mathrm{~d}, 2 \mathrm{H}, J=6.0$ $\mathrm{Hz}, \mathrm{ArH}), 7.68$ (s, 1H, NH), $9.18(\mathrm{~s}, 1 \mathrm{H}, \mathrm{NH}) ;{ }^{13} \mathrm{C}$ NMR (75 MHz, DMSO- $\left.d_{6}\right): \delta 13.93,17.73,19.89,31.79,50.71$, 53.12, 66.35, 99.32, 113.64, 127.43, 136.88, 148.22, 152.00, 158.49, 165.78; IR (KBr): $v_{\max } 3342,3225,3080,1694$, 1627, 1459, 1241, $1126 \mathrm{~cm}^{-1}$; HRMS: $m / z(\mathrm{M}+\mathrm{H})^{+}$calculated for $\mathrm{C}_{17} \mathrm{H}_{22} \mathrm{~N}_{2} \mathrm{O}_{4}: 319.1658$, found: 319.1679 .

Methyl 4-(4-(Isopentyloxy)phenyl)-6-methyl-2-oxo-1,2, 3,4-tetrahydropyrimidine-5-carboxylate (4j) $\mathrm{C}_{18} \mathrm{H}_{24} \mathrm{~N}_{2} \mathrm{O}_{4}$ Recrystallization from ethanol afforded $1.49 \mathrm{~g}$ (90\%) 4j as a white solid. Mp 171-173 ${ }^{\circ} \mathrm{C} ;{ }^{1} \mathrm{H}$ NMR (300 $\left.\mathrm{MHz}, \mathrm{DMSO}-d_{6}\right): \delta 0.94\left(\mathrm{~d}, 6 \mathrm{H}, J=6.0 \mathrm{~Hz}, \mathrm{CH}\left(\mathrm{CH}_{3}\right)_{2}\right)$, 1.62-1.68 (m, $\left.2 \mathrm{H} \mathrm{OCH}_{2} \mathrm{CH}_{2}\right), 1.78-1.87\left(\mathrm{~m}, 1 \mathrm{H}, \mathrm{CH}_{2} \mathrm{CH}\right)$, $2.32\left(\mathrm{~s}, 3 \mathrm{H}, \mathrm{CH}_{3}\right), 3.62\left(\mathrm{~s}, 3 \mathrm{H}, \mathrm{OCH}_{3}\right), 3.62(\mathrm{t}, 2 \mathrm{H}, J=6.0$ $\left.\mathrm{Hz}, \mathrm{OCH}_{2}\right), 5.35(\mathrm{~s}, 1 \mathrm{H}, \mathrm{PhCH}), 6.22(\mathrm{~s}, 1 \mathrm{H}, \mathrm{NH})$, 6.77-6.88 (m, 3H, ArH), 7.18-7.26 (m, 1H, ArH), 8.82 (s, $1 \mathrm{H}, \mathrm{NH}) ;{ }^{13} \mathrm{C}$ NMR $\left(75 \mathrm{MHz}, \mathrm{DMSO}-d_{6}\right): \delta 17.48,23.79$, $24.91,40.09,50.60,53.96,66.41,100.17,113.41,127.46$, 136.51, 148.77, 152.16, 158.29, 165.73; IR (KBr): $v_{\max } 340$, 3231, 3076, 1699, 1620, 1455, 1228, $1139 \mathrm{~cm}^{-1}$; HRMS: 
$m / z(\mathrm{M}+\mathrm{H})^{+}$calculated for $\mathrm{C}_{18} \mathrm{H}_{24} \mathrm{~N}_{2} \mathrm{O}_{4}: 333.1814$, found: 333.1823 .

Methyl 6-Methyl-4-(4-((4-methylbenzyl)oxy)phenyl)2-oxo-1,2,3,4-tetrahydropyrimidine-5-carboxylate (4k) $\mathrm{C}_{21} \mathrm{H}_{22} \mathrm{~N}_{2} \mathrm{O}_{4}$

Recrystallization from ethanol afforded $1.66 \mathrm{~g}(91 \%)$ 4k as a white solid. Mp $250-252{ }^{\circ} \mathrm{C}$; ${ }^{1} \mathrm{H}$ NMR $(300 \mathrm{MHz}$, DMSO- $\left.d_{6}\right): \delta 2.25\left(\mathrm{~s}, 3 \mathrm{H}, \mathrm{CH}_{3}\right), 2.30\left(\mathrm{~s}, 3 \mathrm{H}, \mathrm{PhCH}_{3}\right), 3.53$ $\left(\mathrm{s}, 3 \mathrm{H}, \mathrm{OCH}_{3}\right), 5.01\left(\mathrm{~s}, 2 \mathrm{H}, \mathrm{OCH}_{2}\right), 5.12(\mathrm{~s}, 1 \mathrm{H}, \mathrm{PhCH})$, 6.80-6.90 (m, 4H, ArH), 7.18-7.31 (m, 4H, ArH), $7.76(\mathrm{~s}$, $1 \mathrm{H}, \mathrm{NH}), 9.24(\mathrm{~s}, 1 \mathrm{H}, \mathrm{NH}) ;{ }^{13} \mathrm{C}$ NMR $(75 \mathrm{MHz}, \mathrm{DM}-$ SO- $\left.d_{6}\right): \delta 17.29,20.37,50.67,53.72,70.29,100.09,115.26$, $127.70,128.70,129.71,134.81,135.94,136.78,148.92$, $152.50,158.28,165.49$; IR (KBr): $v_{\max } 3337,3250,3041$, 1693, 1617, 1469, 1240, $1125 \mathrm{~cm}^{-1}$; HRMS: $m / z(\mathrm{M}+\mathrm{H})^{+}$ calculated for $\mathrm{C}_{21} \mathrm{H}_{22} \mathrm{~N}_{2} \mathrm{O}_{4}: 367.1658$, found: 367.1679 .

Methyl 4-(3-(Benzyloxy)phenyl)-6-methyl-2-oxo-1,2, 3,4-tetrahydropyrimidine-5-carboxylate (4l) $\mathrm{C}_{20} \mathrm{H}_{20} \mathrm{~N}_{2} \mathrm{O}_{4}$ Recrystallization from ethanol afforded $1.56 \mathrm{~g}(89 \%)$ $4 \mathrm{l}$ as a white solid. Mp $196-198{ }^{\circ} \mathrm{C} ;{ }^{1} \mathrm{H}$ NMR $(300 \mathrm{MHz}$, DMSO- $\left.d_{6}\right): \delta 2.26\left(\mathrm{~s}, 3 \mathrm{H}, \mathrm{CH}_{3}\right), 3.52\left(\mathrm{~s}, 3 \mathrm{H}, \mathrm{OCH}_{3}\right), 5.07$ $\left(\mathrm{s}, 2 \mathrm{H}, \mathrm{OCH}_{2}\right), 5.10(\mathrm{~s}, 1 \mathrm{H}, \mathrm{PhCH}), 6.95(\mathrm{~d}, 2 \mathrm{H}, J=6.0 \mathrm{~Hz}$, ArH), $7.14(\mathrm{~d}, 2 \mathrm{H}, J=9.0 \mathrm{~Hz}, \mathrm{ArH}), 7.32-7.42(\mathrm{~m}, 5 \mathrm{H}$, $\mathrm{ArH}$ ), 7.73 (s, 1H, NH), 9.23 (s, 1H, NH); ${ }^{13} \mathrm{C}$ NMR (75 MHz, DMSO- $\left.d_{6}\right): \delta 17.20,50.41,53.67,69.87,100.66$, $111.47,112.57,118.49,127.16,127.70,128.76,129.57$, $136.58,147.13,149.32,152.56,159.31,165.25$; IR (KBr): $v_{\max } 3300,3227,3065,1700,1626,1453,1250,1129 \mathrm{~cm}^{-1}$; HRMS: $m / z(\mathrm{M}+\mathrm{H})^{+}$calculated for $\mathrm{C}_{20} \mathrm{H}_{20} \mathrm{~N}_{2} \mathrm{O}_{4}$ : 353.1501, found: 353.1512 .

Methyl 4-(2-Methoxy-4-phenethoxyphenyl)-6-methyl2-oxo-1,2,3,4-tetrahydropyrimidine-5-carboxylate (4m) $\mathrm{C}_{22} \mathrm{H}_{24} \mathrm{~N}_{2} \mathrm{O}_{5}$

Recrystallization from ethanol afforded $1.68 \mathrm{~g}(85 \%)$ 4m as a white solid. Mp 193-195 ${ }^{\circ} \mathrm{C} ;{ }^{1} \mathrm{H}$ NMR $(300 \mathrm{MHz}$, DMSO- $\left.d_{6}\right): \delta 2.24\left(\mathrm{~s}, 3 \mathrm{H}, \mathrm{CH}_{3}\right), 3.03(\mathrm{t}, 2 \mathrm{H}, J=6.0 \mathrm{~Hz}$, $\left.\mathrm{PhCH}_{2}\right), 3.54\left(\mathrm{~s}, 3 \mathrm{H}, \mathrm{OCH}_{3}\right), 3.74\left(\mathrm{~s}, 3 \mathrm{H}, \mathrm{PhOCH}_{3}\right), 4.07$ $\left(\mathrm{t}, 2 \mathrm{H}, J=6.0 \mathrm{~Hz}, \mathrm{OCH}_{2}\right), 5.26(\mathrm{~s}, 1 \mathrm{H}, \mathrm{PhCH}), 5.99(\mathrm{~s}, 1 \mathrm{H}$, $\mathrm{NH})$, 6.68-6.75 (m, 3H, ArH), 7.13-7.28 (m, 5H, ArH), $8.52(\mathrm{~s}, 1 \mathrm{H}, \mathrm{NH}) ;{ }^{13} \mathrm{C}$ NMR $\left(75 \mathrm{MHz}, \mathrm{DMSO}-d_{6}\right): \delta 17.45$, $34.98,50.25,53.30,54.07,69.96,100.56,101.25,115.20$, $126.56,127.66,128.51,128.62,135.47,137.174,148.96$, $152.88,157.07,158.08,164.93$; IR (KBr): $v_{\max } 3350,3215$, 3027, 1698, 1635, 1477, 1241, $1117 \mathrm{~cm}^{-1}$; HRMS: $\mathrm{m} / z(\mathrm{M}+$ $\mathrm{H})^{+}$calculated for $\mathrm{C}_{22} \mathrm{H}_{24} \mathrm{~N}_{2} \mathrm{O}_{5}: 397.1763$, found: 397.1775 .

Methyl 4-(4-(4-(1,3-Dioxoisoindolin-2-yl)butoxy)phenyl)- 6-methyl-2-oxo-1,2,3,4-tetrahydropyrimidine-5carboxylate $(4 \mathrm{n}) \mathrm{C}_{25} \mathrm{H}_{25} \mathrm{~N}_{3} \mathrm{O}_{6}$

Recrystallization from ethanol afforded $2.17 \mathrm{~g}(94 \%)$ 4n as a white solid. Mp $134-136{ }^{\circ} \mathrm{C}$; ${ }^{1} \mathrm{H}$ NMR $(300 \mathrm{MHz}$, DMSO- $\left.d_{6}\right): \delta 1.82$ (br s, $\left.4 \mathrm{H}, \mathrm{OCH}_{2} \mathrm{CH}_{2} \mathrm{CH}_{2}\right), 2.31(\mathrm{~s}, 3 \mathrm{H}$, $\left.\mathrm{CH}_{3}\right), 3.64\left(\mathrm{~s}, 3 \mathrm{H}, \mathrm{OCH}_{3}\right), 3.73-3.75\left(\mathrm{~m}, 2 \mathrm{H}, \mathrm{NCH}_{2}\right)$,
3.93-3.95 (m, 2H, $\left.\mathrm{OCH}_{2}\right), 4.94(\mathrm{~s}, 1 \mathrm{H}, \mathrm{PhCH}), 6.34(\mathrm{~s}, 1 \mathrm{H}$, $\mathrm{NH}), 6.71(\mathrm{~d}, 2 \mathrm{H}, J=9.0 \mathrm{~Hz}, \mathrm{ArH}), 7.15(\mathrm{~d}, 2 \mathrm{H}, J=9.0 \mathrm{~Hz}$, ArH), 7.70-7.83 (m, 4H, ArH), $8.70(\mathrm{~s}, 1 \mathrm{H}, \mathrm{NH}) ;{ }^{13} \mathrm{C} \mathrm{NMR}$ $\left(75 \mathrm{MHz}, \mathrm{DMSO}-d_{6}\right): \delta 17.63,29.41,32.46,37.77,50.37$, $52.95,67.00,100.05,113.32,123.13,127.30,132.33,133.92$, 135.05, 148.76, 152.37, 158.44, 165.72, 168.15; IR (KBr): $v_{\max } 3346,3229,3050,1700,1642,1466,1240,1124 \mathrm{~cm}^{-1}$; HRMS: $m / z(\mathrm{M}+\mathrm{H})^{+}$calculated for $\mathrm{C}_{25} \mathrm{H}_{25} \mathrm{~N}_{3} \mathrm{O}_{6}$ : 464.1822, found: 464.1850 .

Methyl 4-(4-(4-(1,3-Dioxoisoindolin-2-yl)butoxy)phenyl)-6-methyl-2-thioxo-1,2,3,4-tetrahydropyrimidine-5-carboxylate (4o) $\mathrm{C}_{25} \mathrm{H}_{25} \mathrm{~N}_{3} \mathrm{O}_{5} \mathrm{~S}$

Recrystallization from ethanol afforded $2.20 \mathrm{~g}(92 \%)$ 4o as a white solid. Mp $130-132{ }^{\circ} \mathrm{C} ;{ }^{1} \mathrm{H}$ NMR $(300 \mathrm{MHz}$, DMSO- $\left.d_{6}\right): \delta 1.89-1.96\left(\mathrm{~m}, 2 \mathrm{H}, \mathrm{NCH}_{2} \mathrm{CH}_{2}\right), 2.22(\mathrm{~s}, 3 \mathrm{H}$, $\left.\mathrm{CH}_{3}\right), 2.40-2.44\left(\mathrm{~m}, 2 \mathrm{H}, \mathrm{OCH}_{2} \mathrm{CH}_{2}\right), 3.50\left(\mathrm{~s}, 3 \mathrm{H}, \mathrm{OCH}_{3}\right)$, 3.91-3.94 (m, 2H, $\left.\mathrm{NCH}_{2}\right), 4.01-4.07\left(\mathrm{~m}, 2 \mathrm{H}, \mathrm{OCH}_{2}\right), 5.06$ $(\mathrm{s}, 1 \mathrm{H}, \mathrm{PhCH}), 6.56(\mathrm{~d}, 2 \mathrm{H}, J=6.0 \mathrm{~Hz}, \mathrm{ArH}), 6.83(\mathrm{~d}, 2 \mathrm{H}, J$ $=6.0 \mathrm{~Hz}, \operatorname{ArH}), 7.10(\mathrm{~d}, 2 \mathrm{H}, J=6.0 \mathrm{~Hz}, \operatorname{ArH}), 7.33(\mathrm{~d}, 2 \mathrm{H}$, $J=6.0 \mathrm{~Hz}, \mathrm{ArH}), 7.69(\mathrm{~s}, 1 \mathrm{H}, \mathrm{NH}), 9.18(\mathrm{~s}, 1 \mathrm{H}, \mathrm{NH}) ;{ }^{13} \mathrm{C}$ NMR (75 MHz, DMSO- $\left.d_{6}\right): \delta 17.88,30.23,32.89,37.93$, $50.54,53.21,67.25,100.34,113.80,123.36,127.41,132.62$, 134.02, 135.12, 147.88, 158.54, 156.69, 168.24, 174.10; IR $(\mathrm{KBr}): v_{\max } 3380,3260,3029,1647,1452,1185,1137 \mathrm{~cm}^{-1}$; HRMS: $m / z(\mathrm{M}+\mathrm{H})^{+}$calculated for $\mathrm{C}_{25} \mathrm{H}_{25} \mathrm{~N}_{3} \mathrm{O}_{5} \mathrm{~S}$ : 480.1593, found: 480.1620 .

Methyl 4-(4-(4-(4-Allyl-2-methoxyphenoxy)butoxy) phenyl)-6-methyl-2-oxo-1,2,3,4-tetrahydropyrimidine5-carboxylate (4p) $\mathrm{C}_{27} \mathrm{H}_{32} \mathrm{~N}_{2} \mathrm{O}_{6}$

Recrystallization from ethanol afforded $2.18 \mathrm{~g}(91 \%)$ 4p as a white solid. Mp $145-147^{\circ} \mathrm{C} ;{ }^{1} \mathrm{H}$ NMR $(300 \mathrm{MHz}$, DMSO- $d_{6}$ ): $\delta 1.91$ (br s, $\left.4 \mathrm{H}, \mathrm{OCH}_{2} \mathrm{CH}_{2} \mathrm{CH}_{2}\right), 2.27$ (s, $3 \mathrm{H}$, $\left.\mathrm{CH}_{3}\right), 3.25\left(\mathrm{~d}, 2 \mathrm{H}, \mathrm{J}=6.0 \mathrm{~Hz}, \mathrm{PhCH}_{2}\right), 3.55\left(\mathrm{~s}, 3 \mathrm{H}, \mathrm{OCH}_{3}\right)$, $3.76\left(\mathrm{~s}, 3 \mathrm{H}, \mathrm{OCH}_{3}\right), 3.92-4.01\left(\mathrm{~m}, 4 \mathrm{H}, 2 \mathrm{OCH}_{2}\right), 4.97-5.04$ $\left(\mathrm{m}, 2 \mathrm{H},=\mathrm{CH}_{2}\right), 5.27(\mathrm{~s}, 1 \mathrm{H}, \mathrm{PhCH}), 5.82-5.95(\mathrm{~m}, 1 \mathrm{H}$, $=\mathrm{CH}), 6.62(\mathrm{~d}, 2 \mathrm{H}, J=6.0 \mathrm{~Hz}, \mathrm{ArH}), 6.73-6.76(\mathrm{~m}, 3 \mathrm{H}$, $\mathrm{ArH}), 7.13-7.19$ (m, 3H, NH, ArH), $8.11(\mathrm{~s}, 1 \mathrm{H}, \mathrm{NH}) ;{ }^{13} \mathrm{C}$ NMR $\left(75 \mathrm{MHz}\right.$, DMSO- $\left.d_{6}\right): \delta 17.83,30.01,32.56,39.91$, $50.72,53.28,55.83,67.09,68.83,100.08,111.97,112.58$, $113.78,115.57,120.68,127.25,132.67,136.89,137.92$, 148.00, 148.31, 149.64, 152.06, 158.50, 165.85; IR (KBr): $v_{\max } 3350,3200,3046,1694,1625,1448,1223,1165 \mathrm{~cm}^{-1}$; HRMS: $m / z(\mathrm{M}+\mathrm{H})^{+}$calculated for $\mathrm{C}_{27} \mathrm{H}_{32} \mathrm{~N}_{2} \mathrm{O}_{6}$ : 481.2339, found: 481.2365 .

Methyl 4-(3-(4-(4-Allyl-2-methoxyphenoxy)butoxy) phenyl)-6-methyl-2-oxo-1,2,3,4-tetrahydropyrimidine-5-carboxylate (4q) $\mathrm{C}_{27} \mathrm{H}_{32} \mathrm{~N}_{2} \mathrm{O}_{6}$

Recrystallization from ethanol afforded $2.09 \mathrm{~g}(87 \%)$ 4q as a white solid. Mp $141-143{ }^{\circ} \mathrm{C} ;{ }^{1} \mathrm{H}$ NMR $(300 \mathrm{MHz}$, DMSO- $\left.d_{6}\right): \delta 1.84\left(\mathrm{br} \mathrm{s}, 4 \mathrm{H}, \mathrm{OCH}_{2} \mathrm{CH}_{2} \mathrm{CH}_{2}\right), 2.24(\mathrm{~s}, 3 \mathrm{H}$, $\left.\mathrm{CH}_{3}\right), 3.29\left(\mathrm{~m}, 2 \mathrm{H}, \mathrm{PhCH}_{2}\right), 3.53\left(\mathrm{~s}, 3 \mathrm{H}, \mathrm{OCH}_{3}\right), 3.72(\mathrm{~s}$, $\left.3 \mathrm{H}, \mathrm{OCH}_{3}\right), 3.96-3.99\left(\mathrm{~m}, 4 \mathrm{H}, 2 \mathrm{OCH}_{2}\right), 5.00-5.11(\mathrm{Com}-$ plex, $\left.3 \mathrm{H}, \mathrm{PhCH},=\mathrm{CH}_{2}\right), 5.90-5.96(\mathrm{~m}, 1 \mathrm{H},=\mathrm{CH})$, 
6.67-6.87 (m, 6H, ArH), 7.22 (s, 1H, ArH), 7.74 (s, 1H, $\mathrm{NH}), 9.22$ (s, $1 \mathrm{H}, \mathrm{NH}) ;{ }^{13} \mathrm{C}$ NMR $\left(75 \mathrm{MHz}, \mathrm{DMSO}-d_{6}\right): \delta$ 17.78, 30.06, 32.51, 39.87, 50.73, 53.37, 55.82, 67.04, 68.79, $100.16,111.75,111.80,112.44,112.61,115.59,118.77$, $120.67,129.71,132.62,138.03,146.91,148.03,148.35$, $149.61,152.05,159.40,165.72$; IR (KBr): $v_{\max } 3347,3215$, 3081, 1697, 1613, 1463, 1228, $1170 \mathrm{~cm}^{-1}$; HRMS: $m / z(\mathrm{M}+$ $\mathrm{H})^{+}$calculated for $\mathrm{C}_{27} \mathrm{H}_{32} \mathrm{~N}_{2} \mathrm{O}_{6}$ : 481.2339, found: 481.2370 .

\section{Methyl 4-(3-(4-(4-Chlorophenoxy)butoxy)phenyl)-} 6-methyl-2-oxo-1,2,3,4-tetrahydropyrimidine-5-carboxylate (4r) $\mathrm{C}_{23} \mathrm{H}_{25} \mathrm{ClN}_{2} \mathrm{O}_{5}$

Recrystallization from ethanol afforded $1.97 \mathrm{~g}(89 \%)$ 4r as a white solid. Mp $162-164{ }^{\circ} \mathrm{C} ;{ }^{1} \mathrm{H}$ NMR $(300 \mathrm{MHz}$, DMSO- $d_{6}$ ): $\delta 1.83$ (br s, $4 \mathrm{H}, \mathrm{OCH}_{2} \mathrm{CH}_{2} \mathrm{CH}_{2}$ ), $2.22(\mathrm{~s}, 3 \mathrm{H}$, $\left.\mathrm{CH}_{3}\right), 3.51\left(\mathrm{~s}, 3 \mathrm{H}, \mathrm{OCH}_{3}\right), 3.99\left(\mathrm{~m}, 4 \mathrm{H}, 2 \mathrm{OCH}_{2}\right), 5.08(\mathrm{~s}$, $1 \mathrm{H}, \mathrm{PhCH}), 6.75-6.81(\mathrm{~m}, 3 \mathrm{H}, \mathrm{ArH}), 6.93(\mathrm{~d}, 2 \mathrm{H}, J=6.0$ $\mathrm{Hz}, \mathrm{ArH}), 7.20(\mathrm{~d}, 1 \mathrm{H}, J=5.4 \mathrm{~Hz}, \mathrm{ArH}), 7.28(\mathrm{~d}, 2 \mathrm{H}, J=6.0$ $\mathrm{Hz}, \mathrm{ArH}), 7.73(\mathrm{~s}, 1 \mathrm{H}, \mathrm{NH}), 9.21(\mathrm{~s}, 1 \mathrm{H}, \mathrm{NH}) ;{ }^{13} \mathrm{C} \mathrm{NMR}$ $\left(75 \mathrm{MHz}, \mathrm{DMSO}-d_{6}\right): \delta 17.85,28.54,29.64,50.60,53.20$, $65.50,66.86,100.13,111.65,112.43,116.10,118.46,125.52$, $129.38,129.72,147.06,148.36,152.28,157.98,159.37$, 165.72; IR (KBr): $v_{\max } 3360,3200,3044,1700,1625,1450$, 1231, 1164, $758 \mathrm{~cm}^{-1}$; HRMS: $\mathrm{m} / z(\mathrm{M}+\mathrm{H})^{+}$calculated for $\mathrm{C}_{23} \mathrm{H}_{25} \mathrm{ClN}_{2} \mathrm{O}_{5}: 445.1530$, found: 445.1549 .

Methyl 4-(3-(4-(4-Chloro-3-methylphenoxy)butoxy) phenyl)-6-methyl-2-oxo-1,2,3,4-tetrahydropyrimidine5-carboxylate (4s) $\mathrm{C}_{24} \mathrm{H}_{27} \mathrm{ClN}_{2} \mathrm{O}_{5}$

Recrystallization from ethanol afforded $1.99 \mathrm{~g}(87 \%)$ 4s as a white solid. Mp $170-172{ }^{\circ} \mathrm{C} ;{ }^{1} \mathrm{H}$ NMR $(300 \mathrm{MHz}$, DMSO- $d_{6}$ ): $\delta 1.84$ (br s, $\left.4 \mathrm{H}, \mathrm{OCH}_{2} \mathrm{CH}_{2} \mathrm{CH}_{2}\right), 2.24(\mathrm{~s}, 3 \mathrm{H}$,
$\left.\mathrm{CH}_{3}\right), 2.27\left(\mathrm{~s}, 3 \mathrm{H}, \mathrm{PhCH}_{3}\right), 3.52\left(\mathrm{~s}, 3 \mathrm{H}, \mathrm{OCH}_{3}\right), 3.99(\mathrm{~m}$, $\left.4 \mathrm{H}, 2 \mathrm{OCH}_{2}\right), 5.10(\mathrm{~s}, 1 \mathrm{H}, \mathrm{PhCH}), 6.77-6.82(\mathrm{~m}, 4 \mathrm{H}, \mathrm{ArH})$, 6.94 (s, 1H, ArH), 7.20-7.28 (m, 2H, ArH), 7.75 (s, 1H, $\mathrm{NH}), 9.23(\mathrm{~s}, 1 \mathrm{H}, \mathrm{NH}) ;{ }^{13} \mathrm{C} \mathrm{NMR}\left(75 \mathrm{MHz}, \mathrm{DMSO}-d_{6}\right): \delta$ $17.88,19.87,28.48,30.00,50.74,53.39,65.51,67.20$, $100.00,111.40,112.57,114.32,114.47,118.69,129.23$, $129.70,130.68,133.32,147.06,148.38,152.30,155.52$, $159.19,165.61$; IR $(\mathrm{KBr}): v_{\max } 3400,3250,3083,1697$, 1618, 1472, 1230, 1169, $750 \mathrm{~cm}^{-1}$; HRMS: $m / z(\mathrm{M}+\mathrm{H})^{+}$ calculated for $\mathrm{C}_{24} \mathrm{H}_{27} \mathrm{ClN}_{2} \mathrm{O}_{5}$ : 459.1687, found: 459.1708 .

\section{Results and Discussion}

To perform the efficient synthesis of DHPMs, our initial effort was focused on finding the optimized reaction conditions. Thus, the three-component reaction of urea, methyl acetoacetate and benzaldehyde in the presence of CSSNH was selected as the sample reaction to afford methyl 6-methyl-2-oxo-4-phenyl-1,2,3,4-tetrahydropyrimidine-5-carboxylate (4a). Then, the effects of diverse parameters like type of solvent and temperature were studied on the reaction progress (Table 1). Performing the reaction in the absence of solvent is one of the significant aspects of green chemistry. In this regard, the synthesis of DHPM 4a was studied under solvent-free condition. As shown in Table 1 , when the reaction was carried out at room temperature, only $57 \%$ of 4 a was obtained after prolonging the reaction time up to $12 \mathrm{~h}$ (entry 1 ). Regarding to imperative role of the temperature on the reaction progress, we examined the solvent-free synthesis of $\mathbf{4 a}$ at different temperatures (Table 1, entries 1-7). Remarkably, raising the reaction tem-

Table 1. The effect of solvents and temperature for synthesis of $4 \mathrm{a}^{\mathrm{a}}$

\begin{tabular}{|c|c|c|c|c|}
\hline \multirow[b]{2}{*}{ Entry } & \multirow{2}{*}{$+\mathrm{H}_{2} \mathrm{~N}$} & \multirow{2}{*}{ Temperature $\left(^{\circ}\right.$} & $\underset{\left.{ }^{\circ} \mathrm{C}\right)}{\longrightarrow}$ & \multirow[b]{2}{*}{ Yield $^{b}(\%)$} \\
\hline & & & Time (h) & \\
\hline 1 & solvent-free & r.t. & 12 & 57 \\
\hline 2 & solvent-free & 70 & 7 & 83 \\
\hline 3 & solvent-free & 80 & 4.5 & 86 \\
\hline 4 & solvent-free & 90 & 3 & 91 \\
\hline 5 & solvent-free & 100 & 2 & 96 \\
\hline 6 & solvent-free & 110 & 2 & 96 \\
\hline 7 & solvent-free & 120 & 2 & 94 \\
\hline 8 & $\mathrm{MeCN}$ & reflux & 8 & 50 \\
\hline 9 & DMF & 100 & 8 & 45 \\
\hline 10 & toluene & reflux & 10 & 36 \\
\hline 11 & $\mathrm{H}_{2} \mathrm{O}$ & reflux & 5 & 82 \\
\hline 12 & PEG 400 & 100 & 5 & 84 \\
\hline 13 & $\mathrm{EtOH}$ & reflux & 5 & 87 \\
\hline
\end{tabular}

${ }^{\text {a }}$ Reaction condition: benzaldehyde $(5 \mathrm{mmol})$, methyl acetoacetate $(5 \mathrm{mmol})$, urea $(6 \mathrm{mmol}), \mathrm{CSSNH}$ $(0.04 \mathrm{~g}) .{ }^{\mathrm{b}}$ Isolated yield. 
perature resulted in higher yields of $\mathbf{4 a}$ in shorter reaction times. The best result was gained when the synthesis of $\mathbf{4 a}$ was achieved at $100{ }^{\circ} \mathrm{C}$ (entry 5). Practically, further increment of the reaction temperature up to $120^{\circ} \mathrm{C}$ afforded no more considerable improvement in reaction efficacy (entries 6,7$)$. To demonstrate the efficiency of CSSNH under solvent-free condition, the synthesis of $\mathbf{4 a}$ was also achieved using different solvents (Table 1, entries 8-13). Using $\mathrm{MeCN}, \mathrm{DMF}$, and toluene as the solvent affords low to moderate yields of $4 \mathbf{a}$ (entries 8-10). As the results in Table 1 indicate, $\mathrm{H}_{2} \mathrm{O}$, PEG 400 , and $\mathrm{EtOH}$ led to the synthesis of $4 \mathbf{a}$ in $82-87 \%$ yields in longer reaction times (entries 1113). These results confirm the remarkable efficiency of CSSNH for green and solvent-free synthesis of DHPMs.

The catalyst has undeniable role for progress of $3 \mathrm{CR}$ synthesis of DHPMs. Accordingly, the effect of different loaded amounts of CSSNH was evaluated on the progress of the sample reaction (Table 2). As shown in Table 2, in the absence of CSSNH, only $15 \%$ of $4 \mathbf{a}$ was produced even after prolonging the reaction time up to $24 \mathrm{~h}$ (Table 2, entry 1). However, increasing the amount of CSSNH considerably affects the reaction progress (entries 2 and 3 ). The desired DHPM 4a was efficiently obtained when the reac- tion was carried out using $0.04 \mathrm{~g}$ of the catalyst (entry 4). No distinguishable improvement was observed when the amount of CSSNH was enhanced from $0.04 \mathrm{~g}$ up to $0.06 \mathrm{~g}$ (entries 5 and 6). Hence, $0.04 \mathrm{~g}$ of the catalyst was successfully used for synthesis of various DHPMs using CSSNH.

To realize the efficiency and limitations of the present protocol, we compared our obtained data with some previously reported results for synthesis of DHPM $4 \mathbf{a}(\mathrm{Ta}-$ ble 3). As the results in Table 3 demonstrate, the reactions were performed in the presence or absence of solvent at different temperatures. In most methods, the lower yields of $4 \mathbf{a}$ were obtained except entry 7 which uses acidic catalyst in refluxing EtOH. In general, the present method proved to be a smart choice for synthesis of DHPM derivatives. The activity of SSA, and chitosan alone was also compared with that of CSSNH using optimized reaction conditions (Table 3, entries 8-10). Practically neither parental SSA nor chitosan are as efficient as CSSNH for solvent-free 3CR synthesis of DHPMs.

After optimizing the reaction conditions, we explored the versatility and generality of the present method for synthesis of structurally diverse 3,4-dihydropyrimidine-2(1H)-one/thione derivatives. Accordingly, 3CRs be-

Table 2. The effect of catalyst amount for synthesis of $\mathbf{4 a} .^{a}$

\begin{tabular}{lccc}
\hline & & & \\
& & & \\
Entry & CSSNH (X g) & Time (h) & Yield $^{\mathbf{b}}(\%)$ \\
\hline 1 & - & 24 & 15 \\
2 & 0.02 & 7 & 61 \\
3 & 0.03 & 4 & 83 \\
4 & 0.04 & 2 & 96 \\
5 & 0.05 & 1.8 & 93 \\
6 & 0.06 & 2 & 96 \\
\hline
\end{tabular}

${ }^{a}$ Reaction conditions: benzaldehyde $(5 \mathrm{mmol})$, methyl acetoacetate $(5 \mathrm{mmol})$, urea (6 $\mathrm{mmol}), \mathrm{CSSNH}(\mathrm{X} \mathrm{g}) .{ }^{\mathrm{b}}$ Isolated yield.

Table 3. Synthesis of 4 a under various conditions.

\begin{tabular}{|c|c|c|c|}
\hline Entry Ref. & Catalyst and conditions & Time (min) & Yield $^{a}(\%)$ \\
\hline $1^{33}$ & GO-Chitosan, solvent-free, $110^{\circ} \mathrm{C}$ & 10 & 86 \\
\hline $2^{47}$ & {$[$ Btto $][p$-TSA $]$, solvent-free, $90^{\circ} \mathrm{C}$} & 30 & 89 \\
\hline $3^{24}$ & Dendrimer-PWA ${ }^{\mathrm{n}}$, solvent-free, $80^{\circ} \mathrm{C}$ & 40 & 91 \\
\hline $4^{24}$ & Dendrimer- $\mathrm{PWA}^{\mathrm{n}}, \mathrm{EtOH}, 50^{\circ} \mathrm{C}$, ultrasound irradiation & 10 & 90 \\
\hline $5^{48}$ & Isopolyoxomolybdate, solvent-free, $110^{\circ} \mathrm{C}$ & 19 & 92 \\
\hline $6^{13}$ & [TEAPS] $\mathrm{H}_{2} \mathrm{PMo}_{12} \mathrm{O}_{40}$, solvent-free, $105^{\circ} \mathrm{C}$ & 15 & 91 \\
\hline $7^{26}$ & SSA, EtOH, reflux & 360 & 96 \\
\hline $8^{-}$ & SSA, solvent-free, $100^{\circ} \mathrm{C}$ & 120 & 83 \\
\hline $9^{-}$ & Chitosan, solvent-free, $100^{\circ} \mathrm{C}$ & 120 & 79 \\
\hline $10^{\text {This work }}$ & $\mathrm{CSSNH}$, solvent-free, $100^{\circ} \mathrm{C}$ & 120 & 96 \\
\hline
\end{tabular}

\footnotetext{
${ }^{a}$ Isolated yield.
} 
Table 4. Synthesis of $4 \mathrm{a}-\mathrm{s}$ using CSSNH. ${ }^{\mathrm{a}}$ Continued on next page

\begin{tabular}{|c|c|c|c|c|}
\hline Entry Ref. & Product $^{b}$ & Product Number & Time (h) & Yield $^{\mathrm{c}}(\%)$ \\
\hline $1^{26}$ & & $4 a$ & 2 & 96 \\
\hline $2^{49}$ & & $4 b$ & 2 & 94 \\
\hline $3^{49}$ & & $4 c$ & 2.5 & 88 \\
\hline 4 & & $4 d$ & 2.5 & 86 \\
\hline $5^{49}$ & & $4 e$ & 2 & 93 \\
\hline $6^{18}$ & & $4 f$ & 2 & 91 \\
\hline 7 & & $4 g$ & 2 & 89 \\
\hline 8 & & $4 h$ & 2.5 & 87 \\
\hline 9 & & $4 i$ & 2 & 92 \\
\hline 10 & & $4 j$ & 2 & 90 \\
\hline 11 & & $4 \mathrm{k}$ & 2.5 & 91 \\
\hline
\end{tabular}




\section{Continued}

\begin{tabular}{lcccc}
\hline Entry $^{\text {Ref. }}$ & Product Number & Time (h) & Yield $^{\mathbf{c}}$ (\%) \\
\hline 12 & 41 & 2.5 & 89 \\
13 & $4 \mathbf{m}$ & & \\
\end{tabular}

14<smiles>COC(=O)C1=C(C)NC(=O)NC1c1ccc(OCCCCN2C(=O)c3ccccc3C2=O)cc1</smiles>

15<smiles>COC(=O)C1=C(C)NC(=S)NC1c1ccc(OCCCCN2C(=O)c3ccccc3C2=O)cc1</smiles>

16<smiles>C/C=C/Cc1ccc(OCCCCOc2ccc(C3NC(=O)NC(C)=C3C(C)=O)cc2)c(OC)c1</smiles>

17<smiles>C=CCc1ccc(OCCCCOc2cccc(C3NC(=O)NC(C)=C3C(=O)OC)c2)c(OC)c1</smiles>

18<smiles>COC(=O)C1=C(C)NC(=O)NC1c1cccc(OCCCCOc2ccc(Cl)cc2)c1</smiles>

19

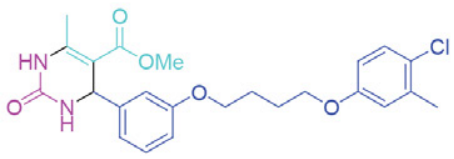

$4 n$

40

$4 p$

$\mathbf{4 q}$

$4 r$

$4 s$
2.5

2.5

2.5

2.5

2.5

2.5
94

92

91

87

89

87

\footnotetext{
${ }^{a}$ Reaction condition: benzaldehyde $(5 \mathrm{mmol})$, methyl acetoacetate $(5 \mathrm{mmol})$, urea $(6 \mathrm{mmol}), \mathrm{CSSNH}(0.04 \mathrm{~g}), 100^{\circ} \mathrm{C} .{ }^{\mathrm{b}}$ All products were characterized by different techniques. ${ }^{\mathrm{c}}$ Isolated yield. ${ }^{\mathrm{d}}$ Two equivalents of urea and methyl acetoacetate were used.
}

tween (thio)urea, methyl acetoacetate and different aldehydes were carried out in the presence of CSSNH under the solvent-free condition at $100^{\circ} \mathrm{C}$ to produce the desired products $\mathbf{4 a}-\mathbf{s}$ in good to excellent yields and also in short reaction times (Table 4 ).

Obviously, CSSNH proved to be a suitable, green, and heterogeneous nano catalyst that effectively catalyzes the synthesis of dihydropyrimidine derivatives 4 . As shown in Table 4, current protocol also works well with different functional groups present in the structure of al- dehydes. In addition, the sterically hindered ortho-substituted aldehydes were successfully converted to the corresponding dihydropyrimidine derivatives in short reaction times and with satisfactory yields (Table 4, entries 3, 4, and 13). The reaction of terephthalaldehyde with 2 equivalents of urea and methyl acetoacetate afforded the desired product $\mathbf{4 h}$ in $87 \%$ yield (Table 4 , entry 8 ). In addition to commercially available aldehydes, several synthetic aldehydes were also employed to synthesize functionalized DHPMs as potential chemotherapeutic agents. These aldehydes 
were pre-synthesized via $\mathrm{S}_{\mathrm{N}} 2$-type reaction of the appropriate hydroxybenzaldehydes with proper alkyl halides. The biological activities of the synthesized compounds are currently under investigation and will be reported in due course.

The recyclability and reusability of the catalyst is another important feature of a green and environmentally friendly method for organic transformations. Thus, the reusability of CSSNH was investigated by its application in five consecutive reactions of model substrates under optimized conditions. To this end, after achieving the first run of 4a synthesis, CSSNH was separated from the reaction mixture using a sintered glass funnel, washed continuously with $\mathrm{EtOH}(2 \times 5 \mathrm{~mL})$, and dried in a vacuum oven at 50 ${ }^{\circ} \mathrm{C}$ for $2 \mathrm{~h}$. The recycled and recovered catalyst was then applied for the next run while no fresh CSSNH was added to the reaction flask. The result of this study is presented in Figure 3.

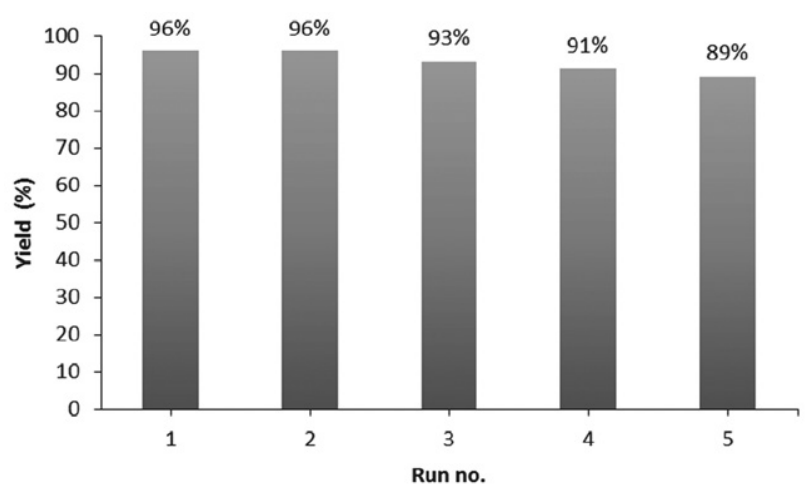

Figure 3. The reusability of CSSNH for synthesis of $\mathbf{4 a}$.

Practically, the ease of recovery and stability of $\mathrm{CSSNH}$ enable its adequate reusability potential. Thus,
CSSNH effectively catalyzes at least five sequential reaction runs while no substantial decline of its catalytic was observed. Moreover, the IR spectrum of reused catalyst after five successive reaction runs is very similar to that of the fresh CSSNH (Figure 4). Apparently, the functionality and nature of CSSNH remain intact which in turn proves the stability of this sustainable and eco-friendly catalyst.

Eventually after elucidating the optimized condition, we were interested to investigate the applicability of this green protocol on a large scale. Consequently, the 3CR of model substrates was conducted on a $70 \mathrm{mmol}$ scale under the optimized conditions which furnished the corresponding DHPM $4 \mathbf{a}$ in $90 \%$ yield.

A plausible mechanism for synthesis of DHPMs using CSSNH as catalyst is outlined in Scheme 2. Hence, the treatment of CSSNH with aldehyde $\mathbf{1}$ affords the activated intermediate I which undergoes the nucleophilic addition of (thio)urea to form intermediate II, followed by dehydration and formation of the acyl imine intermediate III. ${ }^{50}$ Simultaneously, activation of $\beta$-ketoester 3 using CSSNH affords the enol tautomer 3 ' as the nucleophilic species to attack the acyl imine intermediate III which results in the ureide intermediate IV. Subsequent intramolecular cyclization of intermediate IV followed by dehydration of intermediate $\mathbf{V}$ in the presence of CSSNH affords the corresponding DHPM 4. It is worth mentioning that, beside the presence of numerous active ammonium residues in the structure of catalyst, there are plenty of other distributed functional groups such as amine and hydroxyl on the surface of CSSNH that can activate the substrates and intermediates through the hydrogen bonding interactions. This unique property makes $\mathrm{CSSNH}$ as a good candidate for achieving various organic reactions in the presence of a green and efficient heterogeneous nano catalyst.

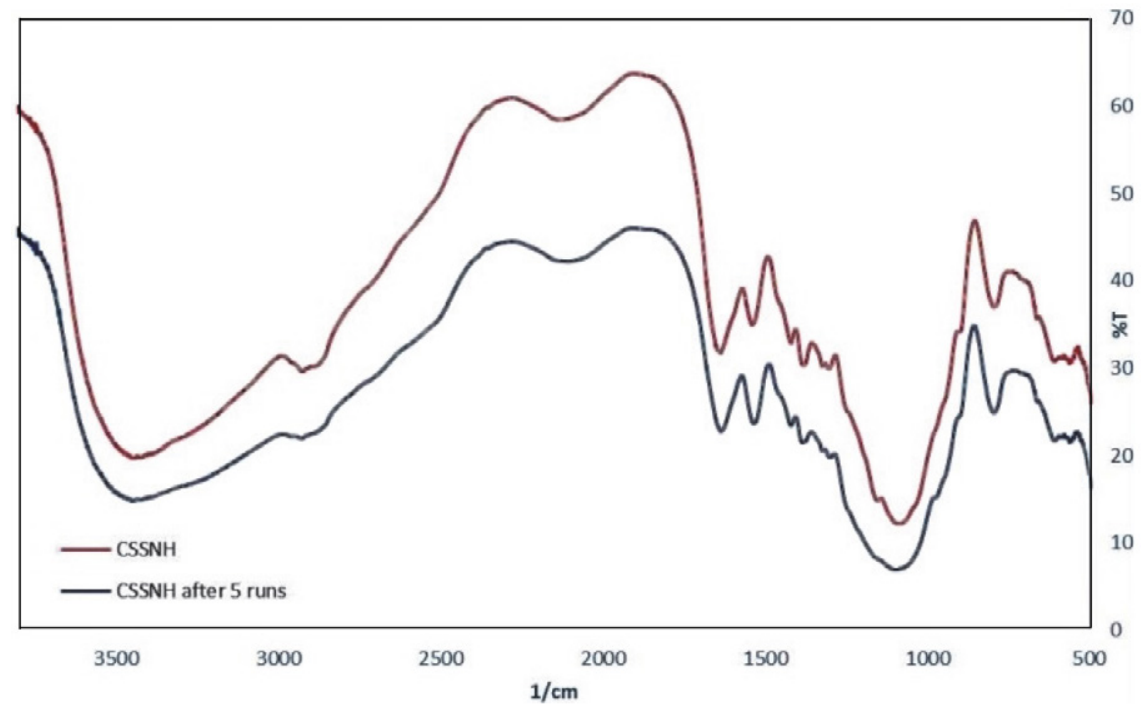

Figure 4. FTIR spectra of fresh CSSNH and recycled CSSNH after five consecutive runs. 


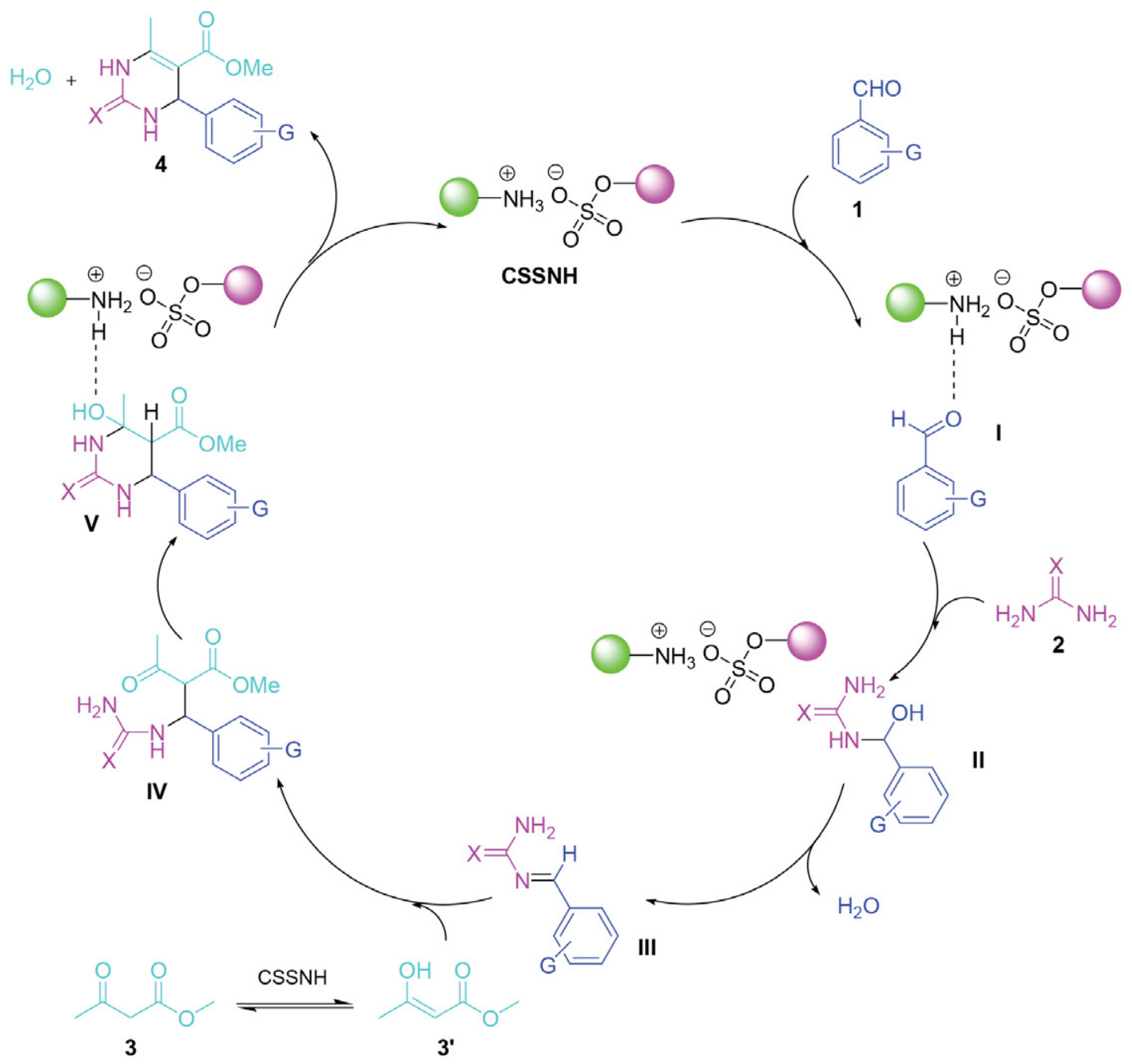

Scheme 2. A plausible mechanism for synthesis of DHPMs using CSSNH.

\section{Conclusion}

In summary, we have described a green and facile protocol based on Biginelli reaction for synthesis of 3,4-dihydropyrimidine-2 $(1 H)$-one/thione derivatives. In this proto$\mathrm{col}$, three-component reaction of commercial and synthetic aldehydes with (thio)urea and methyl acetoacetate under the solvent-free condition in the presence of CSSNH affords the corresponding dihydropyrimidine derivatives in good to excellent yields. The use of a green and heterogeneous nano catalyst, simple operation, cheapness, feasibility on large scale synthesis, elimination of organic solvents and avoidance of strong acidic conditions as well as no need to use toxic transition metals make this method attractive to develop structurally divers DHPMs which are important building blocks in medicinal chemistry and industry.

\section{Acknowledgements}

The authors are grateful to Shiraz University of Technology Research Councils for partial support of this work.

\section{References}

1. K. Tanaka, F. Toda, Chem. Rev. 2000, 100, 1025-1074. DOI:10.1021/cr940089p

2. M. S. Singh, S. Chowdhury, RSC Advances 2012, 2, 4547-4592. DOI: 10.1039/c2ra01056a

3. A. Dömling, W. Wang, K. Wang, Chem. Rev. 2012, 112, 30833135. DOI: $10.1021 / \mathrm{cr} 100233 \mathrm{r}$

4. H. Nagarajaiah, A. Mukhopadhyay, J. N. Moorthy, Tetrahedron Lett. 2016, 57, 5135-5149.

DOI:10.1016/j.tetlet.2016.09.047

5. B. Mohammadi, F. K. Behbahani, Mol. Divers. 2018, 22, 405446. DOI:10.1007/s11030-017-9806-Z

6. A. de Fátima, T.C. Braga, L. da S. Neto, B. S. Terra, B. G. F. Oliveira, D. L. da Silva, L. V. Modolo, J. Adv. Res. 2015, 6, 363-373. DOI:10.1016/j.jare.2014.10.006

7. M. A. Arnold, K. A. Day, S. G. Duron, D. Y. Gin, J. Am. Chem. Soc. 2006, 128, 13255-13260. DOI:10.1021/ja063860+

8. Z. D. Aron, L. E. Overman, J. Am. Chem. Soc. 2005, 127, 3380-3390. DOI:10.1021/ja042875+

9. P. Biginelli, Gazz. Chim. Ital. 1893, 23, 360-413. 
10. B. F. Mirjalili, A. Bamoniri, A. Akbari, J. Iran Chem. Soc. 2011, 8, S135-S140. DOI:10.1007/BF03254290

11. A. Mobinikhaledi, N. Foroughifar, A. Khajeh-Amiri, Reac. Kinet. Mech. Cat., 2016, 117, 59-75.

DOI:10.1007/s11144-015-0931-3

12. A. Farhadi, J. Noei, R. Haji Aliyari, M. Albakhtiyari, M. A. Takassi, Res. Chem. Intermed. 2016, 42, 1401-1406. DOI:10.1007/s11164-015-2092-4

13. H. Peng, Y. Hu, R. Xing, D. Fang, Monatsh. Chem. 2015, 146, 2053-2058. DOI:10.1007/s00706-015-1505-9

14. V. Ramachandran, K. Arumugasamy, S. K. Singh, N. Edayadulla, P. Ramesh, S. K. Kamaraj, J. Chem. Biol. 2016, 9, 31-40. DOI:10.1007/s12154-015-0142-4

15. N. Ahmed, Z. N. Siddiqui, J. Mol. Catal. A Chem. 2014, 387, 45-56. DOI:10.1016/j.molcata.2014.02.019

16. M. Oliverio, P. Costanzo, M. Nardi, I. Rivalta, A. Procopio, ACS Sustainable Chem. Eng. 2014, 2, 1228-1233. DOI:10.1021/sc5000682

17. J. Safaei Ghomi, R. Teymuri, A. Ziarati, Monatsh. Chem. 2013, 144, 1865-1870. DOI:10.1007/s00706-013-1068-6

18. S. P. Bahekar, P. B. Sarode, M. P. Wadekar, H. S. Chandak, J. Saudi Chem. Soc. 2017, 21, 415-419.

DOI:10.1016/j.jscs.2015.09.004

19. J. Ma, L. Zhong, X. Peng, R. Sun, Green Chem. 2016, 18, 1738-1750. DOI:10.1039/C5GC01727K

20. J. T. Starcevich, T. J. Laughlin, R. S. Mohan, Tetrahedron Lett. 2013, 54, 983-985. DOI:10.1016/j.tetlet.2012.12.032

21. Ş. Gülten, J. Heterocyclic Chem. 2017, 54, 1252-1260. DOI: $10.1002 /$ jhet. 2700

22. A. Khorshidi, K. Tabatabaeian, H. Azizi, M. Aghaei-Hashjin, E. Abbaspour-Gilandeh, RSC Adv. 2017, 7, 17732-17740. DOI:10.1039/C7RA00794A

23. J. Safaei-Ghomi, M. Tavazo, G. H. Mahdavinia, Ultrason. Sonochem. 2018, 40, 230-237.

DOI:10.1016/j.ultsonch.2017.07.015

24. Y. Qiu, H. Sun, Z. Ma, W. Xia, J. Mol. Catal. A Chem. 2014, 392, 76-82. DOI:10.1016/j.molcata.2014.04.031

25. R. Javad-Kalbasi, A. R. Massah, B. Daneshvarnejad, Appl. Clay. Sci. 2012, 55, 1-9. DOI:10.1016/j.clay.2011.05.015

26. P. Salehi, M. Dabiri, M. A. Zolfigol, M. A. Bodaghi Fard, Tetrahedron Lett. 2003, 44, 2889-2891.

DOI:10.1016/S0040-4039(03)00436-2

27. M. Maheswara, S. H. Oh, K. T. Kim, J. Y. Do, Bull. Korean Chem. Soc. 2008, 29, 1752-1754.

28. F. Belferdi, F. Bouremmad, S. Shawuti, M. A. Gulgun, Acta Chim. Slov. 2021, in press, http://dx.doi.org/10.17344/acsi.2020.6300

29. R. H. Vekariya, H. D. Patel, Arkivoc 2015, I, 136-159. DOI:10.3998/ark.5550190.p008.975
30. A. Rajack, K. Yuvaraju, Ch. Praveen, Y. L. N. Murthy, J. Mol. Catal. A Chem. 2013, 370, 197-204.

DOI:10.1016/j.molcata.2013.01.003

31. R. Rezaei, S. Malek, M. R. Sheikhi, M. K. Mohammadi, Chem. J. Moldova 2013, 8, 101-106.

DOI:10.19261/cjm.2013.08(2).13

32. J. Lal, S.K. Gupta, D. D. Agarwal, Catal. Commun. 2012, 27, 38-43. DOI:10.1016/j.catcom.2012.06.017

33. A. Maleki, R. Paydar, React. Funct. Polym. 2016, 109, 120-124. DOI:10.1016/j.reactfunctpolym.2016.10.013

34. F. Quignard, A. Choplin, A. Domard, Langmuir 2000, 16, 9106-9108. DOI:10.1021/la000937d

35. H. Honarkar, M. Barikani, Monatsh. Chem. 2009, 140, 14031420. DOI:10.1007/s00706-009-0197-4

36. O. Arjmand, M. Ardjmand, A. M. Amani, M. H. Eikani, Acta Chim. Slov. 2020, 67, 496-506. DOI:10.17344/acsi.2019.5513

37. G. Kravanja, M. Globočnik, M. Primožič, Ž. Knez, M. Leitgeb, Acta Chim. Slov. 2019, 66, 337-343.

DOI:10.17344/acsi.2018.4822

38. Ozyilmaz, A. T. Ozyilmaz, E. I. Bayram, R. H. Akyürekoğlu, Acta Chim. Slov. 2019, 66, 950-957.

DOI:10.17344/acsi.2019.5171

39. S. Behrouz, M. N. Soltani Rad, M. A. Piltan, M. M. Doroodmand, Helv. Chim. Acta 2017, 100, e1700144.

DOI:10.1002/hlca.201700144

40. S. Behrouz, M. N. Soltani Rad, M. A. Piltan, Ultrason. Sonochem. 2018, 40, 517-526.

DOI:10.1016/j.ultsonch.2017.07.046

41. S. Behrouz, M. N. Soltani Rad, M. A. Piltan, Chem. Pap. 2020, 74, 113-124. DOI:10.1007/s11696-019-00863-1

42. S. Behrouz, M. N. Soltani Rad, M. Abdollahzadeh, M. A. Piltan, ChemistrySelect 2020, 5, 7467-7473.

DOI:10.1002/slct.202001722

43. S. Behrouz, J. Saudi Chem. Soc. 2018, 22, 261-268. DOI:10.1016/j.jscs.2016.07.003

44. S. Behrouz, J. Heterocyclic Chem. 2017, 54, 1863-1871. DOI:10.1002/jhet.2777

45. S. Behrouz, J. Saudi Chem. Soc. 2017, 21, 220-228. DOI:10.1016/j.jscs.2016.08.003

46. S. Behrouz, J. Chem. Res. 2016, 40, 540-544. DOI:10.3184/174751916X14709292404728

47. Y. Zhang, B. Wang, X. Zhang, J. Huang, C. Liu, Molecules 2015, 20, 3811-3820.

DOI: $10.3390 /$ molecules20033811

48. A. Nakhaei, A. Davoodnia, S. Yadegarian, Russ. J. Gen. Chem. 2016, 86, 2870-2876. DOI:10.1134/S1070363216120537

49. M. G. Dekamin, F. Mehdipoor, A. Yaghoubi, New J. Chem. 2017, 41, 6893-6901. DOI:10.1039/C7NJ00632B

50. C. O. Kappe, J. Org. Chem. 1997, 62, 7201-7204. 


\section{Povzetek}

V prispevku opisujemo zelen in visoko učinkovit pristop k sintezi 3,4-dihidropirimidin-2(1H)-onskih oz. -tionskih derivatov. Naša strategija vključuje trokomponentno Biginellijevo reakcijo med (tio)sečnino, metil acetoacetatom in aldehidi pod pogoji brez prisotnosti topil ob dodatku hitosan-silikatno sulfatnega nano hibrida (CSSNH) kot zelenega, heterogenega nano katalizatorja. Optimizirani pogoji omogočajo tvorbo ustreznih produktov v kratkih reakcijskih časih z dobrimi do odličnimi izkoristki. CSSNH je cenen, okolju prijazen in nestrupen nano katalizator, ki ga lahko enostavno pripravimo in z njim rokujemo, možna pa je tudi ponovna uporaba brez bistvene izgube aktivnosti. 